\title{
Dilemas éticos expresados por académicos de la Universidad Nacional Autónoma de México
}

\section{Ethical dilemmas expressed by academics from the National Autonomous University of México}

\author{
ANA Hirsch AdLER
}

Como parte del "Estudio sobre la excelencia del profesorado del posgrado de la UNAM", se aplicó una guía de entrevista a 21 académicos de posgrado en 2018. En este artículo se presentan las respuestas a la pregunta abierta: "A lo largo de sus proyectos de investigación, ¿ha enfrentado alguna situación dilemática?”. En la primera parte se incluyen aspectos teóricos sobre los dilemas morales. Los dilemas provienen del enfrentamiento entre concepciones éticas diferentes, procedimientos de especificación y balance, justificación moral, problemas de contenido moral, diversos modelos de actuación, actitudes que se pueden evitar, dilemas de las profesiones y emociones en el desarrollo del juicio moral. En la segunda parte se da cuenta de las doce categorías construidas con base en las respuestas a la pregunta abierta.

As part of the Study about the Excellence of the Graduate Professors in the National Autonomous University of Mexico, we applied an interview guide to 21 academics in 2018. We will present the answers to the question: In your research projects have you encounter dilemmatic situations? In the first part of the article, we included theoretical aspects about what are moral dilemmas?, dilemmas are generated by the confrontation among different ethical conceptions, specification and balance procedures, moral justification, problems of moral content, diverse models to be able to take moral decisions, attitudes that can be avoided, professional dilemmas and emotions in the development of moral judgement. In the second part we present the twelve categories that were constructed from the answers to the open question.
Palabras clave: dilemas éticos, académicos, posgrado, investigación

\section{Keywords:}

ethical dilemmas, academics, graduate programs, research

Recibido: 19 de agosto de 2019 | Aceptado para su publicación: 29 de enero de 2020 Fecha de publicación: 28 de febrero de 2020

Recuperado de: https://sinectica.iteso.mx/index.php/SINECTICA/article/view/1025 doi: 10.31391/S2007-7033(2020)0054-009

* Doctora en Sociología por la UNAM. Investigadora en el Instituto de Investigaciones sobre la Universidad y la Educación de la UNAM. Líneas de investigación: ética profesional, educación y valores, y formación de profesores universitarios. Correo electrónico: hirsch@unam.mx 


\section{INTRODUCCIÓN}

$\mathrm{E}$

1 trabajo es parte del "Estudio sobre la excelencia del profesorado del posgrado de la UNAM", que inició en 2015 y cuenta con la aprobación del Pro-

grama de Apoyo a Proyectos de Investigación e Innovación Tecnológica, de la UNAM, de 2017 a 2019. En 2018 diseñamos una guía de entrevista con diez preguntas abiertas que aplicamos a 21 académicos de las cuatro áreas de conocimiento en las que la institución clasifica sus 41 posgrados. En este artículo, retomamos la pregunta: "A lo largo de sus proyectos de investigación, ¿ha enfrentado alguna situación dilemática?".

El artículo se refiere a algunos aspectos teóricos significativos, por ejemplo, ¿qué son los dilemas morales? Los dilemas provienen del enfrentamiento entre ideales y concepciones éticas diferentes, procedimientos de especificación y balance, justificación moral, problemas de contenido moral, diversos modelos de actuación, actitudes que se pueden evitar, dilemas de las profesiones y emociones en el desarrollo del juicio moral.

También, damos cuenta de las categorías que se construyeron con base en las respuestas a la pregunta por parte de los entrevistados: dilemas sobre el conocimiento, protección de los participantes en las investigaciones, responsabilidad social de los investigadores, plagio y conflictos de interés, presiones por parte de empresas, presiones de las agencias financiadoras, cuestiones sobre género, respeto a la diversidad, conflictos entre los académicos, dificultades respecto a las publicaciones, trámites burocráticos y necesidad de nuevas plazas y problemas con los becarios.

\section{ANTECEDENTES}

El tema que desarrollamos tiene dos antecedentes. En el primero (Hirsch, 2011a), en 2009, diseñamos y aplicamos una guía de entrevista a los 40 coordinadores de posgrado de la UNAM (actualmente hay 41 posgrados) acerca de la ética profesional. Una de las preguntas se refiere a los dilemas éticos que enfrentan los egresados de posgrado en su ejercicio profesional. Recuperamos aspectos centrales del marco teórico acerca de la conceptualización sobre dilemas morales y conductas éticamente inaceptables y cuestionables, y presentamos la clasificación de la información proporcionada por los coordinadores. El segundo (Hirsch, 2011b) concierne a la relevancia de la ética de la docencia a partir de tres aspectos centrales de la ética profesional: dilemas, desafíos y conductas éticas de los profesores.

\section{PRIMERA PARTE: ASPECTOS TEÓRICOS SOBRE LOS DILEMAS MORALES}

\section{¿Qué son los dilemas morales?}

Beauchamp y Childress (2019) los definen como "circunstancias en que las obligaciones morales demandan o parecen demandar que una persona adopte una de dos (o más) acciones alternativas que son incompatibles, de manera que la persona no puede llevar a cabo todas las acciones requeridas" (p. 11). 
Los dilemas provienen del enfrentamiento entre ideales y concepciones éticas diferentes

Para MacIntyre (1983, citado en Jover, 1995), los dilemas provienen del enfrentamiento entre ideales y concepciones éticas diferentes. Considera que "nuestros argumentos morales son retazos que tienen su origen en distintas tradiciones, lo que los hace difícilmente confrontables" (p. 146) y que tienen que ver con la necesidad de adoptar elecciones y decisiones morales. Esto hace que, inevitablemente, tengamos que enfrentar conflictos, desacuerdos y problemas irresolubles. Aunque existen diversas propuestas para reflexionar sobre ellos, de acuerdo con el autor, "carecemos de un criterio claro para su resolución y debemos acostumbrarnos a vivir y trabajar en la incertidumbre moral" (p. 146).

En ello coinciden Beauchamp y Childress (2013), quienes indican que los principios y las reglas en conflicto crean dilemas difíciles. Agregan que son distintos los dilemas morales de los prácticos, ya que estos últimos son conflictos entre los requerimientos morales y el propio interés. Sostienen que los diversos principios morales, reglas y derechos generan conflictos en la vida moral y que, muchas veces, se busca resolver la situación seleccionando una opción sobre otra, aunque ninguna de las acciones es moralmente preferible.

Sobre este punto, Davies (2005) explica que las discusiones de los filósofos acerca de los dilemas morales han estado dominadas por dos preguntas: ¿qué es un dilema moral? y ¿pueden encontrarse dilemas morales genuinos? Estas interrogantes provienen de la existencia de un amplio rango de situaciones en que los individuos enfrentan conflictos irresolubles. Las personas morales están interesadas en este asunto por las severas crisis que pueden tener respecto a ese tipo de cuestiones y a la posibilidad de solucionarlos. Estima que, para poder calificar un dilema como moral, la elección que confronta el individuo necesita estar relacionada con cuestiones moralmente significativas. Ubicarlo como dilema -y no como un problema difícil que puede solucionarse- se basa en la interpretación de cada circunstancia a ser evaluada, como una elección forzosa e ineludible entre dos opciones que son excluyentes una a otra.

\section{Procedimientos de especificación y balance}

Beauchamp y Childress (2013) plantean que, "como ningún teórico moral o código profesional de ética ha presentado exitosamente un sistema de reglas morales libre de conflictos y excepciones" (p. 16), pueden utilizarse con éxito dos procedimientos: especificación y balance. La primera es un proceso que permite reducir la indeterminación de las normas abstractas y, con ello, establecer reglas "con un contenido de acción-guía" (p. 17). La especificación progresiva debe ocurrir para poder manejar la variedad de problemas que surgen, y permitir aminorar los dilemas y conflictos de manera gradual. El balance es el "proceso de encontrar las razones que sustentan las creencias acerca de qué normas morales deben prevalecer" (p. 20). 


\section{Justificación moral}

Beauchamp y Childress (2001) explican que las personas moralmente buenas tienen poca dificultad en hacer juicios correctos. Afirman que las "señales morales" (p. 384) que aprendemos desde temprano en la vida bastan para orientarnos en esas decisiones. Sin embargo, cuando experimentamos "perplejidad o conflicto moral", por lo general, necesitamos una justificación para resolver problemas complejos. Para construirla, se debe establecer el caso y presentar suficientes elementos de sustentación.

Estos autores agregan que en la teoría ética y en la ética biomédica contemporánea hay varios modelos de justificación: el primero parte de una "perspectiva de arriba abajo", que destaca las normas y teorías generales. Beauchamp (2005) complementa lo anterior y agrega que, en los modelos de arriba abajo, la normas generales preexistentes se aplican a una situación nueva y particular, y consiste en aplicar una regla general (ya sean principios, ideales o derechos) a un caso específico.

En cambio, el "modelo de abajo arriba" se centra en la tradición moral, la experiencia y las circunstancias particulares (Beauchamp y Childress, 2013). La pregunta aquí es cómo se toman las decisiones prácticas (Beauchamp, 2005). El autor señala, por ejemplo, acuerdos y prácticas sociales ya existentes, análisis de casos comparativos, relatos de vidas ejemplares, experiencia en casos complejos y la analogía con casos prácticos previos.

El tercer modelo, de acuerdo con Beauchamp y Childress (2001, p. 398) y con Beauchamp (2005, p. 11), tiene que ver con el "equilibrio reflexivo" de John Rawls, que comprende la justificación como una prueba reflexiva de nuestras creencias, principios y postulados teóricos con propósito de hacerlos lo más coherentes posible.

Sin embargo, cuando uno de los rasgos en la teoría moral que sustentamos entra en conflicto con uno o más de los juicios considerados, debemos modificar uno u otro para lograr el equilibrio. Aun los "juicios considerados provisionalmente como puntos fijos" son susceptibles de revisión.

\section{Problemas de contenido moral}

Beauchamp (2005) afirma que la teoría y los principios deben considerarse un suplemento de los casos paradigmáticos, las acciones, la información empírica y la experiencia organizacional, y que hay tres tipos de respuestas en la ética aplicada: la interna, la externa y la mezcla entre ambas: respecto a la primera, el autor retoma a McIntyre (1984, p. 17, citado en Beauchamp, 2005, p. 3) sobre el uso de la práctica para designar un arreglo cooperativo que busca la realización de los bienes internos de la vida comunitaria; la moralidad externa incorpora normas y valores que sostienen, complementan y corrigen los de la moralidad interna (pp. 4-5). Beauchamp (2005) pone como ejemplo de ello a la opinión pública, la ley, las instituciones religiosas y la ética filosófica.

El tercer tipo de enfoque incorpora elementos del internalismo y el externalismo y parte de la convicción de que los compromisos morales se implementan de maneras diversas en las diferentes culturas y grupos. 
Ejemplos de diversos modelos de actuación para tomar buenas decisiones

Cobo (2001) sugiere las siguientes siete pautas:

-Situarse responsablemente ante el problema.

-Identificar bien el caso respecto a las personas, las situaciones, el dilema y las consecuencias de cada alternativa.

- Prever y comparar las consecuencias de cada posible alternativa.

-Evaluarlas moralmente con ayuda de los principios y valores de la ética profesional.

- Contestar las siguientes preguntas: ¿de qué decisión se consiguen más bienes?, ¿ de qué decisión se siguen males menores? y ¿qué pasaría si todos obraran así?

- Decidir u optar en conciencia.

- Asumir la responsabilidad de la decisión.

Francisco Bermejo (2002), de manera similar, sugiere algunos métodos de resolución de casos prácticos, con base en cuatro modelos; tres planteados por otros autores y uno que constituye su propia propuesta metodológica. El primer modelo (López, 1993, citado en Bermejo, 2002) contiene cuatro pasos: analizar con cuidado el contexto general y particular de la situación; centrarse en la naturaleza y las características del tema sobre el que hay que tomar la decisión; considerar el dilema con base en la pregunta ¿cuáles son los derechos que están en juego?; y evaluar las consecuencias previsibles de la decisión sobre las personas, servicios e instituciones.

Loewenberg y Dolgoff (1985, citado en Bermejo, 2002), en el segundo modelo, dan cuenta de un procedimiento de ocho fases: clarificar tres tipos de valores que entran en la situación: sociales, del usuario y del profesional; establecer los fines u objetivos; identificar objetivos alternativos y estrategias de intervención; valorar las posibles consecuencias de cada opción; identificar y ordenar jerárquicamente los principios éticos de cada elección; seleccionar la opción más apropiada; llevarla a cabo, controlar su realización y hacer las correcciones necesarias; y evaluar los resultados y las subsiguientes actividades.

El tercer modelo, de Salcedo (1998, citado en Bermejo, 2002), se refiere a identificar un proceso de siete pasos: aspectos éticos sobre los valores y deberes; individuos, grupos y organizaciones que pueden ser afectados por la decisión ética; posibles actuaciones alternativas junto con los sujetos implicados y los beneficios y riesgos; razones a favor y en contra de cada actuación, que consideran las teorías, principios y guías prácticas éticas, el código de ética y los principios jurídicos, los principios de la profesión y los valores personales (principalmente los que entran en conflicto); consultar con colegas y expertos; tomar la decisión y documentar el proceso que llevó a la toma de decisión; y controlar, evaluar y documentar las consecuencias de la decisión.

El cuarto modelo, planteado por Bermejo (2002), se compone de tres métodos. En el primero hay seis acciones: narrar los hechos; identificar los datos relevantes del caso; identificar la naturaleza del problema (o los problemas) éticos; identificar 
a los afectados por el problema y los motivos de su acción; y generar alternativas posibles al problema planteado y consideración de las dificultades de la puesta en práctica de esas alternativas.

En el segundo método se explica únicamente la valoración ética y la toma de decisión y en el tercero hay solo dos asuntos: realización de la decisión adoptada y evaluación de su puesta en práctica.

Por su parte, Jonsen y Toulmin (1984, citados en Hortal, 2002) sostienen que se necesita:

a) conocer las situaciones concretas con sus circunstancias; b) percibir también las posibilidades que entrañan esas situaciones y los diferentes cursos de acción que en ellas se abren; para poder calibrar el significado de lo que está en juego en esas situaciones y en esos diferentes cursos de acción [...]; c) disponer de una tipología que nos abra los ojos para percibir lo que esa situación tiene de parecido y de diferente con otras situaciones comparables y con otros cursos de acción ya conocidos, sea porque nosotros mismos los hemos vivido o experimentado, o porque pertenecen al acervo común de una cultura, con sus historias, refranes, etc. La riqueza de elementos, perspectivas y facetas que hay que tomar en cuenta es muy importante, pero no basta. Hace falta además: d) capacidad de juicio para ver bajo qué tipologías es adecuado subsumir esa situación y esos diferentes cursos de acción, e) a qué principios hay que apelar [...] y f) cómo se pueden plantear y tal vez resolver los posibles conflictos entre principios, cómo puede justificarse un orden jerárquico entre ellos y si ese orden es estable o cambiante, dependiendo de las circunstancias (p. 105).

Mertzman y Madsen (1999) argumentan que en los dilemas éticos es muy importante formular las preguntas correctas: ¿cuáles son los valores activos en el dilema ético?, ¿cuáles son las virtudes que pueden guiar a un profesional para resolver el dilema ético?, ¿cuáles pueden ser o son las consecuencias de las acciones del profesional?, ¿cuáles son las responsabilidades del profesional? y ¿qué derechos tienen prioridad en la resolución del dilema?

Con todas estas propuestas presentadas a modo de ejemplo, podemos darnos cuenta de la complejidad de los procesos para la toma de decisiones en relación con los dilemas morales. Los modelos son muy parecidos, aunque algunos son más o menos detallados en las facetas o en las preguntas que hay que resolver.

\section{Actitudes que se pueden evitar}

Respecto a la ética profesional, para Bermejo (2002) se deben evitar seis cuestiones:

-La búsqueda de respuestas seguras, simples y cerradas. Esto significa que ante la compleja serie de problemas que conlleva esa ética aplicada, es imposible generar respuestas contundentes y exhaustivas.

-El peso de la experiencia. El autor declara que algunos profesionales consideran suficiente su experiencia para resolver los conflictos, por lo que pueden mostrarse reacios a cualquier tipo de reflexión moral sobre los problemas que los afectan.

-Ética de la situación. Otro problema consiste en centrase únicamente en los casos concretos, y olvidar la importancia de una reflexión más amplia en la que puedan quedar enmarcados los casos particulares. 
-El olvido de las particularidades. El error contrario trata acerca de la abstracción exagerada.

- La centralidad del código. Un problema común es hacer caso dogmáticamente al código profesional.

-El idealismo anticodificador. Este punto es el contrario del tema anterior y se refiere al rechazo a toda normativa ética.

\section{Dilemas que pueden producirse en las profesiones}

Loewenberg y Dolgoff (1985, citados en Bermejo, 2002, pp. 178-180) especifican trece dilemas para los trabajadores sociales, que también son de utilidad para reflexionar sobre otras profesiones. Los autores afirman que pueden darse en la realidad de manera aislada o combinarse. Estos son:

-Conocimiento profesional frente a derechos del usuario. El profesional busca tomar las mejores decisiones en función de su conocimiento y experiencia, pero respeta los derechos de los usuarios de escoger lo que consideran mejor para sí mismos.

-Obligaciones y expectativas en conflicto. El profesional debe tomar sus decisiones tomando en cuenta las obligaciones que tiene en los diversos roles que desempeña.

- Consentimiento informado. La ética profesional requiere el conocimiento informado del usuario, pero estos no siempre entienden todas las implicaciones y consecuencias de las elecciones que se pueden hacer.

-Ambigüedad e incertidumbre. El profesional debe tomar decisiones aun cuando no conozca todas las consecuencias.

- Decir la verdad. Una relación ética demanda que el profesional diga la verdad a sus clientes y usuarios. Sin embargo, hay circunstancias en que decir la verdad puede dañarlos.

- Confidencialidad. No se debe revelar información reservada, pero en algunos casos se demanda al profesional que lo haga.

-Distribución de recursos limitados. Aunque se deben distribuir los recursos de manera equitativa, hay usuarios que necesitan mayor atención que otros.

- Prioridad de los intereses del usuario frente a los del profesional. Se espera que se tomen las decisiones en bien del usuario, pero, en algunos casos, se pueden poner en riesgo los intereses del profesional.

-Suspensión del juicio. Se espera una distinción entre los juicios personales del profesional y los que se ponen en juego en la acción.

-Naturaleza limitada de la relación profesional. Se espera que el profesional tome sus decisiones desde las limitadas relaciones con sus clientes o usuarios, pero algunos requieren una relación más completa para solucionar su problema.

-Escoger el modo más eficaz. Un profesional puede no ser un experto en la modalidad de intervención más eficaz en una determinada situación. 
- Relaciones con los colegas. Se espera de los profesionales que tengan buenas relaciones con sus colegas, pero estas pueden entrar en conflicto con las necesidades de los usuarios.

-Adhesión a los programas y las normas. El profesional debe cumplir las exigencias de la organización que lo contrata, pero algunas de ellas pueden entrar en contradicción con otras de sus obligaciones.

\section{Decisiones éticas y dilemas valorativos}

Callahan (1988) afirma que las preguntas normativas se refieren a los valores y son distintas a las preguntas acerca de los hechos. Los problemas éticos involucran dilemas de valor: "No sólo cuando se refiere a preservar, proteger o buscar un valor o un conjunto de valores, lo que implica el sacrificio de otro valor o conjunto de valores, sino también cuando el no tomar una decisión equivale a decidir a favor de un valor o de un conjunto de valores en competencia" (p. 7).

Esto es similar a lo que indica Altarejos (1998): el no decidir también es decidir, pues muchos conflictos éticos en las profesiones buscan evadirse mediante la abstención de la actuación: "Se puede tener un comportamiento moral, inmoral o incluso amoral, pero no es posible un comportamiento sin sustancia ni referencia moral; cabe la afirmación, la negación o la indiferencia ante la ética: lo que no cabe en su inexistencia” (p. 12).

Para Callahan (1988), los dilemas que surgen en la vida profesional involucran tres tipos de conflictos: entre metas valiosas, entre valores que no consideran las consecuencias y entre principios que demandan buscar resultados deseables. "Mucho de la tarea de construir la propia moralidad reflexiva consiste en clarificar cómo uno cree que se pueden resolver estos conflictos" (Callahan, 1988, pp. 20-21).

\section{Las emociones en el desarrollo del juicio moral}

Retomamos brevemente la propuesta evaluativa y cognitiva en la que se inscribe a Nussbaum (Bedoya, Manacel y Romero, 2015), en la cual las emociones son consideradas como juicios que se basan en creencias y orientan la acción y cumplen un papel esencial en las decisiones. La autora, al definir las emociones como formas de pensamiento o juicios evaluativos que son parte de la vida buena, ofrece un equilibrio al debate de la supremacía de la razón sobre las emociones, al pensar que estas no deben ser eliminadas, controladas o dejadas al margen, ya que son un aspecto esencial y constitutivo de la vida.

Las emociones se conforman por juicios que nos hacen tomar conciencia de nuestra propia humanidad, de las diversas maneras en que somos vulnerables, de nuestra relación con los demás, con lo que consideramos fundamental y relevante para un buen vivir y con aquello que no está dentro de nuestro alcance, lo que demuestra la importancia de otorgar a las emociones un puesto relevante en el campo de la moral. 
Según Bedoya, Manacel y Romero (2015), para Nussbaum, hay cuatro aspectos que caracterizan a las emociones como juicios: que son acerca de algo, esto quiere decir, que tienen objeto; que el objeto es de carácter intencional, esto es, que la emoción corresponde a la percepción e interpretación de quien la experimenta; que encarnan no solo maneras de percibir el mundo, sino que se trata de creencias a menudo muy complejas acerca del objeto; y que poseen un valor o importancia para quien las vive, lo que implica que tienen un carácter local. Incluso se interesan por acontecimientos que tienen lugar a distancia o hechos del pasado.

En suma, a través de las emociones, el sujeto percibe el mundo y son parte constitutiva y esencial del pensamiento, lo que permite establecer una profunda relación entre educar emociones y formar en ética. Nussbaum (2008) afirma que, "en lugar de concebir la moralidad como un sistema de principios que el intelecto imparcial ha de captar y las emociones como motivaciones que apoyan o bien socavan nuestra elección de actuar según esos principios, tendremos que considerar las emociones como parte esencial del sistema de razonamiento ético" (p. 21).

A modo de cierre del apartado teórico, podemos aseverar que la búsqueda de soluciones para aminorar los dilemas éticos es compleja y requiere la reflexión y la evaluación detallada de múltiples acciones y sus posibles consecuencias. Esto se diferencia de los procesos que buscan resolver conflictos éticos, que son más cercanos al trabajo profesional y académico y que pueden subsanarse con menos dificultad. En estos últimos, sin embargo, la reflexión y la evaluación de los problemas son también un aspecto indispensable. Ambos elementos éticos son significativos, pues, cuando se presentan y se reconocen, es porque afectan la actuación de los individuos.

\section{SEGUNDA PARTE: EL TRABAJO EMPÍRICO}

\section{Marco metodológico}

La investigación sobre la excelencia del profesorado del posgrado de la UNAM inició en 2015. Una de las primeras actividades fue la construcción de un marco teórico específico sobre el campo temático, del cual, además, seleccionamos expresamente artículos que contaban con instrumentos de recopilación de información sobre los indicadores de la buena docencia a nivel universitario. Se eligió el "Cuestionario para la selección de indicadores de la excelencia docente universitaria” de Fernández-Cruz y Romero (2010).

Al instrumento de la Universidad de Granada se agregaron cuatro preguntas abiertas: ¿cuáles considera usted que son los cinco valores básicos que la universidad debería promover con sus académicos?; ¿cuáles son los cinco principales rasgos de "ser un buen profesor universitario"?; ¿cómo influyen sus proyectos de investigación en sus actividades de docencia y tutoría?; y mencione hasta tres académicos de posgrado que considere excelentes.

La cuarta pregunta logró conjuntar 710 nombres, de los cuales elegimos algunos profesores e investigadores. En 2018 entrevistamos a 21 de ellos. La guía de aplicó a cinco académicos del área de ciencias físico-matemáticas y de las ingenierías; seis 
de ciencias biológicas, químicas y de la salud; cinco de ciencias sociales; y cinco de las humanidades y las artes. Se trata de once mujeres y diez hombres, en su mayoría, con una amplia trayectoria y antigüedad en la universidad. Todos realizan investigación, además de cumplir con las otras dos funciones sustantivas de docencia y extensión y difusión.

Las entrevistas se transcribieron en su totalidad y se separaron cada una de las diez preguntas abiertas, entre ellas, la de los dilemas éticos. Con las respuestas construimos categorías, con base en la propuesta de Coffey y Atkinson (2003), en cuanto a la identificación de temas y patrones clave: “... Primero, los códigos vinculan diferentes segmentos o ejemplos presentes en los datos. Traemos estos fragmentos de los datos y los reunimos para crear categorías que definimos con base en alguna propiedad o elemento común [...]. También hay un trabajo analítico importante en la identificación de los conceptos pertinentes" (p. 32).

En cada una de las categorías elegimos los testimonios directos que proporcionaron los académicos, y conservamos la vinculación con los posgrados de los que forman parte.

Respuestas a la pregunta abierta: A lo largo de sus proyectos de investigación ¿ha enfrentado alguna situación dilemática?

Es conveniente mencionar que hubo importantes consideraciones por parte de los profesores e investigadores de las cuatro áreas de conocimiento en que la UNAM clasifica sus 41 posgrados. Presentamos a continuación lo que indicaron los académicos sobre las 12 categorías construidas:

\section{- Dilemas sobre el conocimiento}

Se refirieron especialmente a la búsqueda de ideas novedosas, a que existen planteamientos iniciales incorrectos que hay que corregir y que algunas de las ideas que se plantean con fines de investigación ya fueron publicadas por otros. También se habló de la necesidad de incorporar nuevos enfoques a los que ya se utilizan en su propia disciplina. En seguida, algunos testimonios:

Con frecuencia tiene uno una idea que resulta que no es novedosa, eso pasa mucho, hay mucha gente con ingenio en el mundo y pensando lo mismo. Entonces, ya hay que aceptar que así es y consolarse diciendo, bueno, si alguien lo pensó antes, es porque es una buena idea, y luego, muchas veces, se puede decir, pero a lo mejor lo puedo mejorar, lo que es un inicio (entrevista 2, posgrado: Ingeniería).

... sí me he enfrentado a muchos problemas en los cuales el planteamiento inicial fue incorrecto, la estrategia que decidimos fue la incorrecta o los casos típicos, o como les llamo yo, las tristes historias, que alguien hizo un trabajo en el mundo, hizo el trabajo que estamos haciendo o lo publicó antes que nosotros estuviéramos en una posición de poder hacerlo (entrevista 9, posgrado: Ciencias Biomédicas).

... el dilema es cómo puede uno incorporar variables socioculturales a una variable psicológica (entrevista 7, posgrado: Psicología). 
... mis grandes dilemas teóricos son cómo conjuntar la teoría y la práctica (entrevista 19, posgrados: Filosofía, Filosofía de la Ciencias e Ingeniería y Ciencias de la Computación).

Me puse a pensar en esto de la ética, porque yo creo que es importante abrir caminos, cuesta trabajo porque no siempre se entiende lo que se está haciendo, cuando abres un nuevo tema o es un campo que no se reconoce del todo... (entrevista 12, posgrados: Estudios Latinoamericanos y Trabajo Social).

Sobre lo expuesto, consideramos de gran importancia señalar que uno de los valores centrales de la investigación es el de la apertura (openness) (Public Responsibility in Medicine and Research, 2019), que consiste en estar continuamente abierto a nuevas ideas y estrategias. Por un lado, esta visión dinámica del conocimiento implica posibles errores, difíciles elecciones y situaciones inesperadas y, por el otro, genera procesos que contribuyen a la innovación y al avance de todas las disciplinas.

Retomamos la propuesta de Pérez-Pérez y Vázquez (2019) en cuanto a que la sociedad actual experimenta importantes cambios sociales, tecnológicos y económicos y, por ello, los académicos universitarios deben enfrentar situaciones nuevas y competitivas. La globalización y la modernización están creando un mundo cada vez más diverso e interconectado y las tecnologías de la comunicación se diversifican de manera continua, lo que ha generado también un crecimiento exponencial del conocimiento. Los profesores e investigadores necesitan trabajar con una enorme cantidad de información, que debe ser seleccionada, procesada e integrada de acuerdo con los intereses que se persiguen. Para ello se requiere apertura, flexibilidad, capacidad de análisis y la posibilidad de resolver problemas desde perspectivas múltiples.

- Protección de los participantes en las investigaciones

Esta se refiere a la importancia de cumplir con los requisitos acerca del consentimiento informado con todos los sujetos que son parte de las investigaciones, ya que esto permite enfrentar los dilemas, la manera en que se utiliza la información y la protección de los participantes en el trabajo de campo:

... trabajamos con mucha claridad con los participantes [...] siempre incluimos las consideraciones éticas. [...] puede ser una ética muy pactada, a nivel casi de convenio. [...] [se indica] qué se pretende y hasta dónde se llega y se deja muy claro a todos [...] su libertad [para] declinar, [para] no responder y [para] dar por terminado [...] todo eso nos ha ayudado mucho a enfrentar estos dilemas (entrevista 6, posgrado: Psicología).

... tocamos temáticas que pueden afectar el bienestar de las personas [...] si estamos haciendo una investigación, por ejemplo, sobre género y violencia, nuestras investigaciones tienen implicaciones muy profundas [...] el dilema es cómo se utiliza esta información y cómo se puede traducir a una publicación que sea útil para las personas (entrevista 7, posgrado: Psicología).

... en mi trabajo de investigación lo que más problemas me ha causado eran las encuestas sobre el terreno, sobre todo con estudiantes y sobre todo con las mujeres [...] siempre he intentado mandar a los estudiantes en pareja, un compañero y una compañera y nunca a las mujeres solas (entrevista 15, posgrado: Ciencias Políticas y Sociales). 
Resultó de gran interés que, al indagar sobre los dilemas éticos, haya surgido el tema de cómo proceder a fin de proteger a los participantes en las investigaciones. Esta perspectiva forma parte de la ética de la investigación que se lleva a cabo con seres humanos (human research ethics), en la que se plantea la necesidad de resguardar sus derechos y su bienestar, y buscar, al mismo tiempo, la construcción de nuevo conocimiento.

Una de las estrategias más conocidas y utilizadas a nivel mundial es el Código de Núremberg, publicado en 1947, que constituye el primer instrumento internacional sobre esta problemática. El documento plantea el proceso de consentimiento informado, que incorpora dos convicciones éticas: los individuos deben ser tratados como agentes autónomos y las personas con autonomía disminuida tienen derecho a mayor protección. Después de este primer logro, las agencias federales y las asociaciones científicas y profesionales de varios países desarrollaron numerosas regulaciones, que están siempre sujetas a la discusión y al debate, pues exponen diferentes interpretaciones (Shamoo \& Resnik, 2015).

- Responsabilidad social de los investigadores

En cuanto al papel que deben desempeñar los investigadores respecto a la sociedad, se manifestó la necesidad de que los financiamientos beneficien a las personas y de cumplir con la responsabilidad social con base en el trabajo que se desarrolla:

... el dilema podría ser: ¿para quién es la investigación? porque nosotros pertenecemos a una institución pública y cuando conseguimos fondos normalmente son de espacios públicos y [estos deben] servir a la gente y a quien dio los fondos (entrevista 7, posgrado: Psicología).

... hasta hace ocho años, yo sólo estaba por producir artículos para subir mi nivel en el Sistema Nacional de Investigadores y subir en el PRIDE [Programa de Primas al Desempeño del Personal Académico de Tiempo Completo], [...] pero al moverme a Nutrición, me vi frente al dilema de decir, bueno, ahora estoy en el Hospital de México, su objetivo es desarrollar proyectos que beneficien a pacientes, aquí no se trata de conocimiento, el conocimiento viene después, lo importante son los estudios aplicativos. Me costó mucho trabajo [...] cambiar mi entrenamiento, mi manera de pensar y relacionarme ahora con [otro] grupo de individuos. Yo lo hago, porque sí siento una responsabilidad social [...] (entrevista 11, posgrados: Ciencias Médicas, Odontológicas y de la Salud y Ciencias Biomédicas).

Concordamos con Bird (2014) en que la responsabilidad social de los científicos proviene del hecho de que son miembros de la sociedad como un todo y, además, los son de las comunidades científicas a las que pertenecen. Sus capacidades y su formación los prepara para brindar conocimiento especializado y perspectivas para el entendimiento y análisis de temas y problemas que afectan a la sociedad de la que son parte. Sus responsabilidades sociales surgen no solo de que están financiados, directa e indirectamente, por el público, sino que también derivan de que la investigación se lleva a cabo a nombre de la sociedad, con base en las necesidades, intereses y prioridades sociales y de las consecuencias esperadas de sus resultados. 
- Plagio y conflictos de interés

La conducta no ética más mencionada fue la del plagio; también se indicaron conflictos de interés:

... el conflicto más horrible que me ha tocado ha sido con una colega joven, que llegó aquí con un muy buen currículo, justo a interactuar en los temas en que yo estaba empezando a juntarme con otros colegas de aquí del Instituto [...] [el trabajo] al mismo tiempo que lo envían a la revista, se lo envié a ella, [...] para que me comentara que le parecía y ella explotó y dijo que le estábamos plagiando sus ideas y, realmente, nos atacó durísimo. Finalmente, el director pidió la opinión de colegas de otra dependencia que eran expertos en el tema y [...] el consenso fue que no había plagio y lo único que le pedimos es que retirara la acusación y no quiso (entrevista 4, posgrado: Ciencias Físicas).

La parte del plagio [...] siempre aparece [...]. [También hay] dilemas de simulación que es un problema muy serio en la academia, de gente que simula y tiene la desfachatez de exponer [...] el problema frente a un dilema ético, pues es la ética, o sea qué hago yo, lo dejo pasar y que el muchacho siga su vida o el colega o quién sea, o trato de decir que la ciencia y la formación, tienen ciertos requerimientos éticos que son indispensables. Entonces, hay que reprobar, hay que denunciar, hay que sancionar como se pueda (entrevista 14, posgrado: Ciencias Políticas y Sociales).

Cuando yo veo un conflicto de interés, pues lo hago clarísimo y explico por qué declino, o cuáles serían mis razones por las cuales yo me abstendría de participar, y con todos los alumnos de la misma manera, lo primero que yo externo es cuál es mi rol de tutor o de orientador y que los alumnos también están en total libertad de desear cambiar de director de tesis y de venir a pedir una asesoría (entrevista 6, posgrado: Psicología).

Sobre los temas de plagio y conflictos de interés, recuperamos el trabajo de Aluja y Birke (2004), quienes sostienen que hay una diferencia entre las conductas éticamente inaceptables, que se refieren a la violación de códigos, normas o contratos existentes, y las conductas éticamente cuestionables, que tratan acerca de situaciones que no están formalmente establecidas en códigos o normas. En las primeras, podemos citar la fabricación, la falsificación y el plagio. Estos autores definen el plagio como la apropiación de ideas, apropiación de métodos innovadores, datos o cuerpo de un texto como propio sin citar la fuente o reconocer al creador de la idea.

Son muchas las conductas éticamente cuestionables; entre ellas están los conflictos de interés, que son de diversa índole: "En términos generales se considera que un conflicto de interés está representado por todas aquellas acciones o comportamientos, mediante los cuales se obtiene una ganancia personal de manera abusiva, ya sea con el fin de obtener una mejor posición laboral o un beneficio económico personal" (Macrina, 2000, citado en Aluja y Birke, 2004, p. 113).

\section{- Presiones por parte de empresas}

Este tema se trabajó con mayor amplitud en Hirsch (2014), trabajo en el cual incluimos los resultados obtenidos, en 2009, con los coordinadores del posgrado de la UNAM y catorce profesores de tres universidades de Valencia en España, a dos preguntas de una guía de entrevista abierta: ¿cuáles son los dilemas éticos que enfrentan los egresados de posgrado? y ¿qué desafíos está presentando la sociedad a las universidades en cuanto a la formación de los profesionales de su área de conocimiento? 
En 2018 informamos acerca de la solicitud de servicios específicos por parte de algunas empresas que buscan, indebidamente, obtener certificados sin llevar a cabo los procedimientos necesarios:

Llegan a solicitar al Centro algunos servicios, por ejemplo, nos haría el favor de brindar un certificado de que yo empresa A hago el proceso bien y vale [...] ese fabricante me pide ese certificado, sin hacer el proceso, y claro, por supuesto rechazamos eso y [le contestamos] que si quiere el certificado, tiene que permitirnos hacer una serie de pruebas con las que nosotros podemos avalar que su procedimiento está de acuerdo con las normas (entrevista 1, posgrados: Ingeniería y Ciencia e Ingeniería de Materiales).

... al trabajar con industrias [...] y con grandes empresas, es muy frecuente que te sientas presionado por lo que esperan de ti, pero, justamente, aquí hay que estar de una pieza y hacer lo correcto [...] encuentras un resultado que no es lo que esperaba la empresa [....] (entrevista 3, posgrado: Ingeniería Ambiental).

Al respecto de lo expresado por los participantes, coincidimos con la propuesta de Hortal (2002) respecto a que el profesional se legitima como experto y combina dos elementos centrales: la competencia profesional y la responsabilidad: "Los profesionales no lo son sólo por ser expertos capacitados, sino también por estar comprometidos en la prestación de determinados servicios específicos" (p. 25).

- Presiones de las agencias financiadoras

Acerca de la visión cuantitativa del Consejo Nacional de Ciencia y Tecnología (Conacyt), de no considerar que algunos estudiantes de posgrado no puedan obtener el grado en el tiempo requerido por el trabajo complejo que realizan, rescatamos los siguientes testimonios:

... casi todos los posgrados en donde yo estoy involucrado son de excelencia del Conacyt. El Consejo quiere cifras, no les interesa tanto la calidad, por ejemplo, si los estudiantes no se gradúan en cuatro años, ya es un punto menos, si se extendieron tanto tiempo ya es un punto menos, si el tutor tiene pocos estudiantes también [...] lo que tendrían que hacer los posgrados es extender los plazos en los casos excepcionales. Los mejores estudiantes y que publican en revistas importantes, se tardan más tiempo por lo mismo (entrevista 10, 2018, posgrado: Ciencias Bioquímicas).

La visión cuantitativa del anterior testimonio proviene de un modelo dominante, que emplean las instituciones de educación superior y las agencias financiadoras, vinculada al logro de indicadores, como eficiencia, eficacia, rendición de cuentas, evaluación y productividad académica ligada al pago de estímulos económicos (Acosta, 2014). Esta tendencia difícilmente toma en cuenta situaciones especiales.

- Cuestiones sobre género

En esta categoría se ubicó, en especial, la sobrecarga de trabajo, la problemática de las investigadoras de poder combinar su trabajo académico con la vida familiar y los estereotipos de los roles diferenciados entre hombres y mujeres:

... hubo una época en la que me costó mucho trabajo arrancar mi laboratorio, que fue cuando empecé mi posdoctorado, yo vine de una facultad en donde no había muchos recursos y, entonces, me costaba trabajo avanzar. Luego, en esa época tuve a mis hijos [...] y eso también me absorbía mucho tiempo y mucha atención [...]. Tuve que ir muy despacio 
y me costó mucho trabajo publicar y lo fui haciendo [...] Llegué a cuestionarme, si realmente yo era buena para esto y si realmente eso era lo que quería, [...] después poco a poco las cosas se fueron asentando (entrevista 8, posgrado: Ciencias Bioquímicas).

Todo el tiempo me enfrento a dilemas en los momentos de investigación, porque los dilemas tienen que ver, a veces, con la sobrecarga que tenemos las investigadoras. [...] lo he hecho por un compromiso con la investigación y con las mujeres (entrevista 13, posgrados: Ciencias Políticas y Sociales y Psicología).

... yo puedo decir que en algunos momentos fui cuestionada por algunos académicos de la facultad por ser mujer, cuando me nombraron como directora de seminarios; además era muy joven $[y]$ no estaba casada [...] ahí se reproducen roles estereotipados, [...] no puede haber un reconocimiento a las habilidades de uno como mujer, porque hay un cuestionamiento en automático (entrevista 16, posgrado: Derecho).

La figura del profesorado universitario es clave para ir avanzando en la tarea de alcanzar la igualdad efectiva entre mujeres y hombres en las instituciones educativas. Es evidente que, a pesar de los logros producidos, aún hay problemas de inequidad y conductas no éticas que hay que resolver. Recuperamos, como ejemplo, el trabajo de Guil (2007), que define

los Techos de Cristal (Glass Ceiling) como las barreras "invisibles", sutiles que dificultan la promoción de las mujeres y la ocupación de puestos de poder, en cualquier ámbito laboral remunerado. [...] se trata de manera clara de la sutileza e invisibilidad del proceso del que la mayoría de las personas -incluso inicialmente las propias protagonistas-, son ajenas (p. 1).

\section{- Respeto a la diversidad}

Las opiniones presentadas por los académicos tienen que ver con los grupos diversos sexualmente, con el fomento de relaciones armoniosas entre los alumnos y la incorporación de un número creciente de mujeres estudiantes:

Formar en valores es complejo. Tuve una experiencia en el curso de campo. Me aviso mi colega de que se había inscrito una persona que recientemente se había cambiado de sexo. En el curso, nosotros los hospedamos en un lugar y normalmente decimos, "los hombres comparten cuartos y las mujeres comparten cuartos", y yo me quedé con esto, quizás, va a hacer un problema, me espanté y dije, cómo debo reaccionar ante esto, y nos sentamos con mi colega y dijimos, si en realidad esta persona es mujer, dice que es mujer, yo nada más tengo que aceptar eso, y yo espero que las chicas lo acepten igual (entrevista 5, posgrado: Ciencias de la Tierra).

Incluso en comportamiento los alumnos tienen que aprender cómo se llevan unos con otros, [...] el respeto a la diversidad dentro del mismo departamento es algo que fomento mucho. Algo que me ha llamado la atención en los últimos años, de diez solicitudes de ingreso al laboratorio, son ocho o nueve mujeres, o sea que el aspecto del feminismo dentro del laboratorio es bienvenido y muy respetado (entrevista 9, posgrado: Ciencias Biomédicas).

Es relevante que se hubiera hecho mención del tema de la formación en valores, que en la ética profesional y en la ética de la investigación ocupa un lugar destacado, especialmente en los procesos de deliberación sobre los dilemas éticos. Las universidades son lugares de formación y socialización que conjuntan personas con identidades diversas que, en todos los casos, deben ser respetadas. Es sugestivo que los académicos introdujeran esta temática, que, por su importancia, se 
ha integrado en diversas leyes y reglamentos que la reconocen y la defienden. Se constituye en uno de los elementos necesarios para promover la convivencia en el ámbito universitario.

\section{- Conflictos entre los académicos}

Respecto a los profesores e investigadores, se indicaron tres aspectos: la falta de espacios de discusión entre los colegas, el desinterés por el trabajo en equipo y los grupos de poder:

... he discutido con colegas [...] cuando uno abre un tema a la discusión, hay silencio, no expresan lo que piensan, se van por otra parte, y le dejan a uno de hablar, [...] lo ignoran, porque no hay mejor manera de excluir a alguien que no mencionándolo, ni para bien, ni para mal. [...] no he encontrado yo en la UNAM, entre muchos de mis colegas, un espacio de discusión viva y abierta (entrevista 17, posgrado: Historia).

... nadie ya trabaja en equipo, porque la competencia es individual, y quien es coordinador quiere serlo por toda la vida, y no nos dan tiempo, no nos abren un espacio [...]. El crecer en el ámbito académico, también se está volviendo un dilema, porque tú quieres tener cierta protección académica, vas, por un lado; quieres ser independiente, te vas por otro lado, todo tiene un costo. Hay grupos de poder que cada vez se están fortaleciendo, siempre han existido las tribus, sin embargo, pareciera que si uno no da un voto a un estudiante, está uno en contra del profesor o del estudiante y, entonces, empieza a verse una molestia y uno [...] empieza a enfrentar un dilema (entrevista 20, posgrado: Pedagogía).

Resulta significativo que se hayan manifestado problemas de convivencia entre los académicos de algunos posgrados, puesto que este tipo de conductas no éticas generan ambientes de trabajo difíciles y pueden obstaculizar el cumplimento de las funciones sustantivas. Estas situaciones, en general, no se expresan abiertamente. Podemos relacionar este problema con uno de los valores éticos relevantes, como el respeto, en este caso, a los colegas y con los conflictos de interés.

- Dificultades respecto a las publicaciones

Se hizo mención sobre el largo tiempo que tardan los artículos en publicarse; la necesidad de publicar mucho para poder contar con los estímulos económicos de la propia universidad y de agencias financiadoras nacionales, así como la exigencia de publicar en inglés:

... cuando uno somete un artículo mientras de mayor nivel sea la revista, más tiempo toma [su publicación] [...] y eso no se toma en cuenta, ni para el Conacyt, los posgrados, ni para los dineros (entrevista 10, posgrado: Ciencias Bioquímicas).

... para que se me acepte un trabajo, es el gran dilema ahorita, me voy por las treinta hojas y digo lo estrictamente necesario para que se pueda publicar o a un trabajo más reflexivo y más analítico y me lleva tiempo, pero si no, no tengo PRIDE [Programa de Primas al Desempeño del Personal Académico de Tiempo Completo] y no tengo SNI [Sistema Nacional de Investigadores]. [...] hay un problema: ¿para quién estoy escribiendo? para una publicación, que no le va a interesar, porque no nos leemos, o escribo para mis estudiantes, o quiero escribir para mis colegas, para mis profesores, o me meto en una dinámica de escribir y tengo muchos artículos [...] son dilemas que estamos enfrentando (entrevista 20, posgrado: Pedagogía). 
... una de las cosas que ha sucedido como efecto de esas revistas de alto impacto, es que ya no estamos publicando en español. [...] hay maestros, estudiantes y profesionistas que [podrían] leer ese artículo en español y que pueden cambiar su práctica y su formación. [...] el dilema es: ¿qué podríamos hacer para que esta información fuera valorada y también útil para nuestra propia disciplina? (entrevista 7, posgrado: Psicología).

Podemos afirmar que, respecto a las funciones sustantivas de la universidad, se ha priorizado, por parte de muchos de los académicos, el tiempo y esfuerzo que se destina a la investigación, muchas veces en contraposición con los que se proporcionan en las labores de docencia. Esto se debe a los incentivos económicos y de prestigio que acarrea la investigación y la producción en términos de las publicaciones. Lo dicho por los entrevistados da cuenta del esfuerzo que debe hacerse para obtener los beneficios de las agencias financiadoras de la propia universidad y de las de carácter nacional e internacional.

\section{- Trámites burocráticos y necesidad de nuevas plazas}

En los temas anteriores se mostró inconformidad con algunas políticas de la UNAM y del Conacyt. Aquí presentamos testimonios sobre las dificultades con la burocracia y el problema de que se forman buenos estudiantes que no podrán quedarse en la universidad ante la falta de plazas:

... Se desespera uno con la burocracia, en los trámites que hay que hacer aquí (entrevista 2, posgrado: Ingeniería).

Los intereses de los alumnos que vienen aquí son muy diferentes, porque para ser arquitectos no necesitan posgrados. Un egresado de este posgrado [.....] [no es como otros posgrados] en donde le piden un doctorado para seguir investigando; aquí no hay centros de investigación en Arquitectura [....] a los alumnos les cuesta mucho trabajo porque no hay campos de investigación. [.....] hay veinte profesores, que tienen muchísimos años, pero no hay plazas. Se convierte en un círculo vicioso (entrevista 18, posgrado: Arquitectura).

El cumplimiento de las funciones sustantivas de la universidad se complica aún más con los múltiples trámites que deben realizarse, no solo para la propia institución, sino también en cuanto a los diversos informes que hay que llenar para la promoción de proyectos de investigación y para conseguir estímulos de las agencias financiadoras externas. Nos referimos previamente a las condiciones de tiempo y esfuerzo para poder llevar a cabo todas las actividades necesarias, a las que hay que agregar los requisitos de corte académico-administrativo y de evaluación.

- Problemas con los becarios

Los entrevistados señalaron dos cuestiones: el hecho de que muchos de los estudiantes que ingresan a los posgrados no poseen los conocimientos que se demandan y la resistencia de algunos de ellos o ellas para cumplir con los trabajos que se requieren:

... los alumnos no traen la preparación que deben [tener] en el posgrado, llegan [...] tratando de completar conocimientos que no les dieron en la licenciatura, y eso no es un posgrado, el posgrado está a un nivel diferente, y eso les cuesta mucho trabajo a los estudiantes (entrevista 18, posgrado: Arquitectura). 
... cuando los estudiantes tienen beca de la UNAM, sobre todo con extranjeros, hay una cláusula que les exige hacer trabajos para el posgrado, y yo ahí trate de incorporar estudiantes, [...] pero dos de ellos se negaron a hacerlo, diciendo que yo los explotaba y mi argumento era decirle, espérate, primero, existe esta cláusula y, segundo, es parte de tu educación. [...] Tuve problemas con una alumna que me dijo, yo no estoy dispuesta porque la beca de la DGAPA [Dirección General de Asuntos del Personal Académico-UNAM], lo único que me exige es trabajar en mi tesis y le dije, sabes que respeto tu posición, pero aquí vamos a la chamba [...] (entrevista 19, posgrados: Filosofía, Filosofía de la Ciencias e Ingeniería y Ciencias de la Computación).

Las becas son un elemento sobresaliente que contribuye significativamente a la formación de profesionales calificados; sin embargo, los académicos entrevistados indicaron problemas con algunos de los estudiantes, en especial en el cumplimiento de los compromisos que adquieren con las universidades y con el Conacyt. Por ello, consideramos que la formación ética debe estar presente en todos los niveles de la enseñanza, sin exceptuar el del posgrado.

A modo de cierre de la parte empírica, señalamos que la información que proporcionaron los académicos fue muy abundante, lo que nos permitió clasificarla en doce categorías. Se trata de ideas diversas, desde la más importante que se refiere al conocimiento hasta las que citan aspectos relevantes para el trabajo universitario. Asimismo, hay opiniones comunes entre los académicos de las cuatro áreas de conocimiento.

\section{A MODO DE CONCLUSIóN}

La parte teórica sobre los dilemas éticos tiene una finalidad formativa, por los temas de gran relevancia de la ética de la investigación. Los elementos teóricos presentados poseen un elevado nivel de abstracción y complejidad, mientras que las ideas de los académicos se refieren a diversas situaciones problemáticas que han encontrado en su trabajo en la universidad. Sin embargo, los entrevistados demostraron sensibilidad ética y dieron cuenta de procesos reflexivos que utilizaron con el propósito de entender y aminorar los dilemas que enfrentan en su labor cotidiana.

Quedó claro que no todo lo planteado se genera y puede resolverse directamente en el ámbito universitario, pues hay obstáculos que provienen de otras instituciones y organizaciones, como las empresas que utilizan sus servicios, las agencias financiadoras como el Conacyt, que fue mencionado varias veces, y los órganos de publicación nacionales e internacionales.

La pregunta sobre los dilemas éticos nos permitió conocer aspectos interesantes de la universidad y detonó muchas y variadas respuestas, incluso de campos emergentes de investigación, por ejemplo, los asuntos de género y el respeto a la diversidad. Se manifestaron también algunos conflictos que se mantienen, por lo general, en la invisibilidad, como las inadecuadas relaciones entre los académicos, los problemas con los becarios y las conductas no éticas, entre ellas el plagio y los conflictos de interés. 


\section{REFERENCIAS BIBLIOGRÁFICAS}

Acosta, A. (2014). Gobierno y gobernanza de la universidad: el debate emergente. En H. Casanova y R. Rodríguez (coords.). Bordón, Revista de Pedagogía, Madrid, Sociedad Española de Pedagogía, vol. 66, núm. 1, pp. 31-44. doi: 1013042/bordon.2014.66102

Aluja, M. y Birke, A. (2004). Panorama general sobre los principios éticos aplicables a la investigación científica y la educación superior. En M. Aluja y A. Birke. El papel de la ética en la investigación científica y la educación superior (pp. 145180). México: Fondo de Cultura Económica/Academia Mexicana de Ciencias.

Altarejos, F. (1998). La docencia como profesión asistencial. En F. Altarejos, J. A. Ibáñez- Martín, J. A. Jordán y G. Jover. Ética docente: elementos para una deontología profesional (pp. 19-50). Barcelona: Editorial Ariel.

Beauchamp, T. (2005). The nature of applied ethics. En R. G. Frey \& C. Heath (eds.). A companion to applied ethics (pp. 1-15). Reino Unido: Blackwell Publishing Ltd.

Beauchamp, T. \& Childress, J. (2019). Principles of biomedical ethics (8a. ed.). Nueva York: Oxford University Press.

Beauchamp, T. \& Childress, J. (2013). Principles of biomedical ethics (7a. ed.). Nueva York: Oxford University Press.

Beauchamp, T. \& Childress, J. (2001). Principles of biomedical ethics (5a. ed.). Nueva York: Oxford University Press.

Bedoya, N., Manacel, E. y Romero, W. H. (2015). Las emociones en el desarrollo del juicio moral, en perspectiva de educación filosófica. Análisis, Revista Colombiana de Humanidades, vol. 47, núm. 86, pp. 103-118. Recuperado de www.redalyc.org/artiuclo.oa?id=515551481005

Bermejo, F. (2002). La ética del trabajo social. Bilbao, España: Desclée de Brouwer, SA.

Bird, S. (2014) Socially responsible science is more than "good science". JMBE Journal of Microbiology \& Biology Education, vol. 15, núm. 2, pp. 169-172. Recuperado de https: //www.ncbi.nih.gov/pmc/articles/PMC4278471/

Callahan, J. (ed.) (1988). Ethical issues in professional life. Nueva York: Oxford University Press.

Cobo, J. M. (2001). Ética profesional en ciencias humanas y sociales. Madrid, España: Huerga y Fierro Editores, SL.

Coffey, A. y Atkinson, P. (2003). Los conceptos y la codificación. En A. Coffey y P. Atkinson. Encontrar el sentido a los datos cualitativos. Estrategias complementarias de investigación (pp. 31-63). Colombia: Contus, Editorial Universidad de Antioquia.

Davis, A. (2005). Moral dilemmas. En R. G. Frey \& C. Heath (eds.) A companion to applied ethics (pp. 487-497). Reino Unido: Blackwell Publishing Ltd.

Fernández-Cruz, M. y Romero, A. (2010). Indicadores de excelencia docente en la Universidad de Granada. Revista Portuguesa de Pedagogía, vol. 44, núm. 1, pp. 83-117. doi: 10.14195/1647-8614_44-1_4

Guil, A. (2007). Docentes e investigadoras en las universidades españolas: visibilizando techos de cristal. Revista de Investigación Educativa, vol. 25, núm. 1, pp. 11-113. Recuperado de https://www.researchgate.net/publication/41570427_Docentes_e_Investigadoras_en_ las_universidades_españolas_Visibilizando_techos_de-cristal 
Hirsch, A. (2011a). Dilemas éticos que enfrentan los egresados de posgrado de la Universidad Nacional Autónoma de México según los coordinadores de los programas. En A. Hirsch y R. López Zavala (coords.). Ética y valores profesionales. Trece experiencias de investigación universitaria en México (pp. 49-81). Universidad Autónoma de Sinaloa/Universidad Iberoamericana Puebla/Universidad Autónoma de Tamaulipas/Universidad Autónoma del Estado de Morelos/Universidad Autónoma de Yucatán/Universidad de Monterrey.

Hirsch, A. (julio-diciembre, 2011b). Dilemas, tensiones y contradicciones en la conducta ética de los profesores. Sinéctica, Revista Electrónica de Educación, núm. 37. Recuperado de http://www.sinectica.iteso.mx/index. php?cur=37\&art=37_00

Hirsch, A., Navia, C. y Pérez Castro, J. (junio, 2014). Professional ethical dilemmas in universities in Spain and in Mexico. International Journal for CrossDisciplinary Subjects in Education, vol. 5, núm. 2, pp. 1657-1661.

Hortal, A. (2002). Ética general de las profesiones. Bilbao, España: Editorial Desclée de Brouwer, SA.

Jover, G. (1995). Líneas de desarrollo y fundamentación en el campo de la deontología de las profesiones educativas. Teoría de la Educación, Revista Interuniversitaria, vol. 7, pp. 137-152.

Mertzman, R. \& Madsen, P. (1999). Introduction to professional ethics. En Valores para el ejercicio profesional (pp. 119-126). Monterrey: Instituto Tecnológico y de Estudios Superiores de Monterrey, Centro de Valores Éticos.

Nussbaum, M. (2008). Paisajes del pensamiento. La inteligencia de las emociones. Barcelona, España: Ediciones Paidós Ibérica, SA.

Pérez-Pérez, C. y Vázquez, V. (2019). Las universidades en la sociedad del conocimiento: el reto de enseñar a aprender a lo largo de toda la vida. En M. Musaio \& R. Sanz (eds.). Sfide educative e riflessione pedagogica internazionale (pp. 119-133). Milano: EDUCatt.

Public Responsibility in Medicine and Research (2019). Core values. Recuperado de https://www.primr.org/about/mission

Shamoo, A. \& Resnik, D. (2015). Chapter 11: The protection of human subjects in research. En A. Shamoo \& D. Resnik. Responsible conduct of research (pp. 237-282) (3a. ed.). Nueva York: Oxford University Press. 